\title{
Competência física percebida de crianças com paralisia cerebral: Que relações com a percepção dos seus pais e a sua função motora?
}

\author{
Rui Corredeira \\ Adília Silva \\ António M. Fonseca
}

https://doi.org/10.5628/rpcd.08.01.09

\author{
Faculdade de Desporto \\ Universidade do Porto \\ Portugal
}

\section{RESUMO}

Este estudo teve como principal objectivo examinar a relação entre o grau de função motora real de crianças com Paralisia Cerebral, com idades entre os 4 e 9 anos, e a percepção de competência física percebida das mesmas e de seus pais relativamente a elas. Foram utilizadas as versões portuguesas $d a$ Dutch Pictorial Scale of Perceived Competence and Social Acceptance for children with Cerebral Palsy - EPPCASCPPp - a 81 crianças e seus pais, e da Gross Motor Function Measure (versão de 66 itens) - TMFM-66 - às crianças.

Da aplicação do TMFM-66 resultou a divisão das crianças em três grupos de diferente comprometimento motor. A análise dos valores médios relativos à EPPCASCPCp (subescala de competência física), atribuídos pelas crianças e seus pais, permitiu constatar que, de uma forma geral, independentemente do seu comprometimento motor, aquelas apresentavam valores de percepção de competência elevados. Verificouse também que a avaliação que as crianças faziam da sua competência física fora sempre superior à atribuída pelos seus pais, e que os valores médios de competência física percepcionados por ambos, decresciam à medida que aumentava o grau de comprometimento motor avaliado pela TMFM- 66 .

As correlações entre a função motora e a competência física percebida foram moderadas sendo superiores no caso da avaliação por parte dos pais $(\mathrm{r}=0,621)$ comparativamente à percepção manifestada pelas crianças $(r=0,503)$.

Estes resultados sugerem a adequação da utilização da EPPCASCPCp, como indicador indirecto para avaliação de função motora em crianças com paralisia cerebral.

Palavras-chave: paralisia cerebral, competência física percebida, função motora

\section{ABSTRACT \\ Physical Perceived Competence in children with Cerebral Palsy: what relationships with parents perception and their own motor function}

The aim of this study was to investigate the relationship between the actual motor function level of children with cerebral palsy, aged between 4 and 9 years old, their physical self-perceived competence and their parents' when compared to them. The Portuguese version of the Dutch Pictorial Scale of Perceived Competence and Social Acceptance for children with Cerebral Palsy - EPPCASCPPp - was used in 81 children and their parents, and the Portuguese version of the Gross Motor Function Measure (66 items version) - TMFM-66 - was used only for the children.

Three different groups of motor impairment were defined by using the TMFM-66 aplication.

The EPPCASCPCp (physical competence subscale) scores of the children and their parents showed that in general and independently of their motor impairment, those children presented high perceived competence levels. We also found that the children's physical competence evaluation was always higher when comparing to that of their parents, and concomitantly, the average physical competence scores perceived by both decreased as the level of motor impairment assessed by TMFM-66 increased.

The correlations between motor function and physical perceived competence were moderate, but higher when comparing the parents evaluation $(r=0,621)$ to the children's perceptions $(r=0,503)$.

These findings suggest that the EPPCASCPCP is adequate as an indirect pointer of motor function evaluation in cerebral palsy children.

Key-words: cerebral palsy, physical perceived competence, motor function 


\section{INTRODUÇÃO}

Sempre que observamos o desempenho de uma criança numa determinada actividade, seja ela no domínio social, cognitivo, físico ou outro, efectuamos de imediato, um determinado julgamento acerca da competência que ela demonstra na execução da mesma, tendo por base a nossa prévia experiência naqueles domínios.

Porém, o nível de competência que atribuímos à criança que observamos (por exemplo no desempenho de uma actividade de índole motora) não é necessariamente igual ao que ela atribui a si própria, podendo diferir igualmente dos julgamentos efectuados por outros, nomeadamente os pais, professores, treinadores, amigos ou outros. Deste pressuposto imerge a importância de tentarmos perceber que relação existe entre a avaliação da competência real de um indivíduo num determinado domínio e a que é percebida pelo próprio.

Estudos na área das auto-percepções ${ }^{(26,56)}$ forneceram indicadores que sustentam a ideia destas poderem desempenhar um papel preponderante na autoavaliação que a criança faz das suas áreas fortes e fracas, permitindo-lhes o estabelecimento de objectivos realistas e, concomitantemente, o desenvolvimento de estratégias de aprendizagem efectivas e a previsão de resultados com sucesso.

Neste contexto, salientam-se os estudos de Harter(24) e Harter \& Pike (25), defendendo que a competência percebida se constitui como uma variável básica importante para a motivação.

Susan Harter(27), considera a competência percebida como sendo um constructo de cariz multidimensional que conduz o indivíduo a aventurar-se em diferentes contextos de realização, orientados pelos domínio social, físico, cognitivo ou outros. Mais recentemente, temos assistido a um aumento do interesse sobre tópicos relacionados com as autodescrições e as autoavaliações ${ }^{(19,54)}$, nomeadamente no que se refere a populações com necessidades especiais $(27,51)$.

Ao referir-se à forma como cada pessoa se descreve (i.e., às características e atributos reconhecidos pela própria), Harter(27) utiliza o termo autorepresentações ou alternadamente autopercepções ou autodescrições, considerando que a distinção entre elas é um tanto arbitrária e resulta, muitas vezes, apenas de diferentes metodologias utilizadas.
O desenvolvimento da competência percebida depende muito da interacção entre o indivíduo e o meio onde está inserido, assim como do ajuste entre ambos, tendo em conta as múltiplas e diferentes características daqueles (34).

Contudo, quando o âmbito de estudo é alargado a populações com necessidades especiais, como foi o caso deste, incidindo concretamente em crianças com Paralisia Cerebral (PC), deverão ser também tomadas em consideração as características da própria deficiência as quais, conjuntamente com o referido anteriormente, determinarão o significado que o indivíduo atribui à sua condição.

Klein \& Evans ${ }^{(32)}$, acrescentam que quando a intervenção envolve crianças, as autopercepções parecem exercer um determinado efeito (positivo ou negativo) ao nível do seu desempenho sugerindo, ao mesmo tempo, que a percepção de competência, de entre os diferentes constructos no campo das autopercepções, possa ser entendida como a categoria que surge como mais aliciante para os técnicos ligados ao processo de reabilitação de crianças com PC, sendo mesmo parte integrante do processo terapêutico.

Curiosamente, os resultados conhecidos relativos à PC, parecem não ser tão lineares, uma vez que as crianças com esta patologia não se avaliam como menos competentes, concretamente ao nível da competência física $(15,48,49,60)$. Um estudo realizado por Rudisill e colaboradores ${ }^{(44)}$ concluiu que a competência percebida é uma função da competência motora actual e da idade, podendo generalizar-se estes factos a ambos os sexos. Aliás, a este propósito, Goodway e Rudisill(22) afirmam que uma intervenção ao nível da competência motora pode constituir um caminho efectivo para influenciar a competência percebida.

Assim, parece haver uma cada vez maior necessidade de implementação de programas de intervenção muito específicos que tal como referem Ulrich(57) e Welk e colaboradores ${ }^{(61)}$, permitam aumentar o nível de adequação entre a percepção que a criança ou jovem tem das suas competências (neste caso competência física) e as suas competências reais. Recorrendo a uma grande diversidade de instrumentos, alguns estudos $(16,42,44,61)$ têm sido desenvolvidos no âmbito de populações com necessidades especiais, tentando perceber a relação entre a função motora e alguns domínios relacionados com a per- 
cepção de competência.

Nesse sentido, este estudo teve como principal objectivo a avaliação da relação entre o grau de função motora real de crianças com Paralisia Cerebral, com idades entre os 4 e 9 anos, e a percepção de competência física percebida das mesmas e de seus pais, através da utilização da versão portuguesa da Dutch Pictorial Scale of Perceived Competence and Social Acceptance for children with Cerebral Palsy de Vermeer \& Venhof(60) - EPPCASCPCp - adaptada para a nossa população por Corredeira(13) e a versão adaptada por Andrada e Gimenez(2) - TMFM66 - da Gross Motor Function Measure (GMFM-66) de Russel e colaboradores ${ }^{(44)}$.

\section{METODOLOGIA}

\section{Amostra}

Depois de previamente termos solicitado autorização para consulta dos ficheiros relativos às crianças com PC inscritas no Centro de Reabilitação de Paralisia Cerebral do Porto, seleccionamos um total de 134 que tinham idades entre os 4 e 9 anos, eram colaborantes, e apresentavam um Q.I. superior a 70. Este último critério deveu-se ao facto de assim podermos assegurar que todos os participantes tinham capacidade para perceberem todos os itens de um dos instrumentos (EPPCASCPCp) utilizados, tal como sugerido por Vermeer et al.(59) para a sua aplicação. Contactamos os pais dessas crianças através dos terapeutas da equipa que atendiam as crianças em regime de atendimento externo ou, no caso das orientações periódicas, por intermédio de uma carta, onde constavam a explicação e objectivos da pesquisa, bem como a solicitação para participação.

Alguns dados obtidos através da consulta dos dossiers individuais das crianças seleccionadas, foram posteriormente complementados pelo preenchimento, por parte dos pais, de uma ficha relativa a informações gerais de carácter sócio-demográfico e comportamental acerca das crianças e de si mesmos. A amostra final deste estudo foi constituída por 49 rapazes $(60,5 \%)$ e 32 raparigas $(39,5 \%)$ e respectivos pais que autorizaram a participação e colaboração no mesmo.

Do total das crianças, $35(43,2 \%)$ tinham idades entre os 4 e os 6 anos e $46(56,8 \%)$ entre os 7 e os 9 anos. Em termos de diagnóstico funcional, 54 $(66,7 \%)$ das crianças apresentavam marcha independente (ambulatórias), sendo as restantes $27(33,3 \%)$ dependentes de terceiros ou de ajudas técnicas para o seu deslocamento (não ambulatórias ).

Em relação ao grau de afectação motora das crianças (i.e., classificação topográfica), verificámos que o maior número de crianças foi constituído pelas diplégicas $(39,5 \%)$, seguido dos hemiplégicos $(33,3 \%)$ e ainda dos tetraplégicos $(27,2 \%)$.

\section{Instrumentos}

Para a recolha de dados foram utilizados dois instrumentos: a versão portuguesa da Dutch Pictorial Scale of Perceived Competence and Social Acceptance for children with Cerebral Palsy DPSPCSACCP de Vermeer \& Venhof(61) - EPPCASCPCp - adaptada para a nossa população por Corredeira(13) e a versão adaptada por Andrada e Gimenez(2) - TMFM-66 - da Gross Motor Function Measure (GMFM-66) de Russel e colaboradores(44).

\section{A Escala Pictórica de Percepção de Competência e Aceitação Social em Crianças com Paralisia Cerebral (versão portuguesa)}

A EPPCACCPCp é uma escala de aplicação específica para crianças com PC com idades entre os 4 e os 9 anos, composta por um total de 40 itens distribuídos por 4 subescalas de 10 itens cada uma: duas relativas às competências física e cognitiva percebidas e outras duas relativas à aceitação social percebida pelos pais e pelos pares.

Neste estudo apenas aplicamos os itens referentes à subescala de competência física. Esta escala no seu conjunto, prevê versões distintas relativamente à idade, sexo e à capacidade de locomoção da criança (i.e., se a criança é ambulatória, ou seja, se tem marcha independente ou se, pelo contrário, é não ambulatória, ou seja, dependente de terceiros ou de ajudas técnicas para a sua locomoção).

O protocolo de aplicação desta escala propõe instruções específicas que incluem a administração individual, a apresentação em plano vertical das imagens (inibindo assim défices perceptivos) e a descrição oral das mesmas. Solicita-se à criança que se identifique com a imagem com a qual se considera mais semelhante e, posteriormente, que indique o grau de semelhança, seleccionando os círculos grandes ou mais pequenos que representam, respectivamente, "muita semelhança" ou "pouca semelhança". O valor relativo a cada subescala é calculado através da média 
dos valores atribuídos aos 10 itens de cada uma delas, sendo que, a cada item é atribuída uma pontuação de 1 a 4 pontos, onde 1 corresponde a não muito competente/socialmente aceite, consoante a subescala de que se trate até 4 , situação que traduz que a criança é muito competente/socialmente aceite, igualmente de acordo com a subescala avaliada. A aplicação deste instrumento variou entre 20 a 45 minutos por criança, tendo sido realizada individualmente no Centro de Paralisia Cerebral do Porto e sempre pelo mesmo investigador, especialmente treinado para o efeito, respeitando as instruções definidas para a sua aplicação. O mesmo inquiridor aplicou a escala aos pais, os quais responderam acerca de características dos filhos, em momentos distintos mas no mesmo dia, tendo a aplicação a duração média de 15 minutos.

\section{A TMFM-6}

A TMFM-66 é um instrumento padronizado que se destina a medir de forma qualitativa as mudanças na função motora global de crianças ao longo do tempo. A TMFM- 66 compreende 66 itens, todos eles contemplando a função motora global de uma criança com 5 anos sem qualquer tipo de comprometimento(47). Os itens desta escala são agrupados em 5 dimensões, da seguinte forma: i) decúbitos e rolamentos (4 itens); ii) sentar (15 itens); iii) gatinhar e ajoelhar (10 itens); iv) posição de pé (13 itens) e v) andar correr e saltar (24 itens).

A pontuação é atribuída com base na observação do desempenho da criança relativamente a cada item, tendo estes uma pontuação entre o (zero) e 3 pontos, consoante os seguintes desempenhos da criança: i) não consegue iniciar a actividade (zero pontos); ii) inicia sem ajuda: corresponde a $10 \%$ da execução de um item completo num teste de 0 a $100 \%$ (1 ponto); iii) completa parcialmente: considera-se que a execução da função está entre $10 \%$ e $100 \%$ da realização do item ( 2 pontos); iii) completa de forma independente: considera-se que a execução da função é de $100 \%$ (3 pontos).

Através da aplicação deste instrumento, obtêm-se diferentes pontuações para cada uma das cinco dimensões motoras avaliadas e uma pontuação total, correspondente à medida global da função motora das crianças que pode variar entre $0 \%$ e $100 \%$.
Com base nesta aferição, e de acordo com um estudo de Palisano e colaboradores ${ }^{(40)}$, onde foi feita uma aproximação da máxima pontuação prevista da TMFM-66, para diferentes graus de severidade motora da PC, dividiram-se as crianças em três grupos, a considerar:

i) Grupo I: crianças que obtiveram uma pontuação superior a $89 \%$;

ii) Grupo II: crianças que obtiveram uma pontuação igual ou superior a $61 \%$ e inferior a $89 \%$;

iii) Grupo III: crianças que obtiveram uma pontuação inferior a $61 \%$.

A duração média de aplicação deste teste foi de 45 minutos, tendo sido realizada individualmente a cada uma das crianças no centro de Paralisia Cerebral do Porto, por duas terapeutas com experiência no uso deste instrumento.

\section{Análise estatística dos dados}

As proporções foram comparadas recorrendo ao teste do Qui-quadrado $\left(\chi^{2}\right)$, com correcção de Yates quando o valor esperado em algumas das células era inferior a 20. Quando esse valor era inferior a cinco, utilizou-se aprova exacta de Fischer.

Como as variáveis quantitativas contínuas na amostra deste estudo, referentes à avaliação da percepção de competência física, não satisfaziam os requisitos de uma distribuição normal, a comparação das mesmas foi realizada pelo recurso a testes não paramétricos. Foi utilizado o teste de Kruskal-Wallis, para comparação das grandezas por grupos da função motora. A comparação dos valores médios de competência física percebida pelas crianças e pelos respectivos pais foi realizada recorrendo ao teste de Wilcoxon, para amostras emparelhadas.

Para avaliar a interdependência entre as medidas efectuadas por diferentes métodos (EPPCASCPCp e TMFM-66) recorreu-se ao coeficiente de correlação de Spearman.

Avaliou-se ainda a concordância na classificação dos indivíduos por tercis de distribuição das variáveis função motora e competência física percebida, através da percentagem de concordência de indivíduos classificados no mesmo tercil e através do coeficiente Kappa.

A análise estatística foi realizada recorrendo ao software SPSS ${ }^{\circledR}$ versão 15. 
Quadro 1. Descrição da amostra por grupos relativos à função motora (TMFM-66) e de acordo com características das crianças.

\begin{tabular}{|c|c|c|c|c|}
\hline & Grupo I & Grupo II & Grupo III & \\
\hline & $n[\%]$ & $n[\%]$ & $n[\%]$ & $p$ \\
\hline \multicolumn{5}{|l|}{ Sexo } \\
\hline Feminino & $14(43,8)$ & $9(28,1)$ & $9[28,1)$ & \\
\hline Masculino & $32(65,3)$ & $10(20,4)$ & $>(14,3)$ & 0,139 \\
\hline \multicolumn{5}{|l|}{ Idade } \\
\hline$<$ Panos & $14(42,4)$ & $12(36,4)$ & $7(21,2)$ & \\
\hline$\geq 7$ anos & $32(66,7)$ & $7(14,6)$ & $9(18,8)$ & 0,049 \\
\hline \multicolumn{5}{|l|}{ Residência } \\
\hline Rural & $16(57,7)$ & $9(19,2)$ & $8(23,1)$ & \\
\hline Urbana & $30(55,2)$ & $10(31,0)$ & $8(13,8)$ & 0,379 \\
\hline \multicolumn{5}{|l|}{ Escolaridade Pais } \\
\hline Até $9^{\circ}$ ano & $33(62,3)$ & $10(18,9)$ & $10(18,9)$ & \\
\hline$>9^{\circ}$ ano & $13(46,4)$ & $9(32,1)$ & $6(21,4)$ & 0,325 \\
\hline \multicolumn{5}{|l|}{ Capacidade de locomoção } \\
\hline Com marcha independente & $45(84,9)$ & $8(15,1)$ & $0(0)$ & \\
\hline Sem marcha independente & $1(3,6)$ & $11(39,3)$ & $16(57,1)$ & $<0,001$ \\
\hline \multicolumn{5}{|l|}{ Classificação topográfica } \\
\hline Tetraplegia & $9(40,9)$ & $5(22,7)$ & $8(36,4)$ & \\
\hline Diplegia & $12(37,5)$ & $12(37,5)$ & $8(25,0)$ & \\
\hline Hemiplegia & $25(92,6)$ & $2(7,4)$ & $0(0)$ & 0,800 \\
\hline \multicolumn{5}{|l|}{ Actividade desportiva } \\
\hline Com prática & $33(66,0)$ & $9(18,0)$ & $8(16,0)$ & \\
\hline Sem prática & $12(40,0)$ & $10(33,3)$ & $8(26,7)$ & 0,075 \\
\hline
\end{tabular}

\section{RESULTADOS}

O quadro 1 descreve a distribuição da amostra pelos 3 grupos definidos a partir da avaliação da função motora global efectuada a partir da TMFM-66, e de acordo com características demográficas, sociais, clínicas e comportamentais das crianças avaliadas.

A distribuição percentual das crianças não variou significativamente por sexo, idade, área de residência, escolaridade dos pais, classificação topográfica e a prática de actividade desportiva. Como esperado a percentagem de indivíduos não ambulatórios foi significativamente superior nos indivíduos do grupo II e grupo III, ou seja aqueles que manifestam maior comprometimento da função motora.

A análise dos valores médios das respostas relativos à EPPCASCPCp, concretamente no que se refere à sua subescala de percepção de competência física, atribuídos pelas crianças e seus pais em cada um dos grupos da função motora global, permite verificar uma grande amplitude de resposta, com os valores a oscilarem entre $1,68(\mathrm{dp}=0,33)$ e $3,52(\mathrm{dp}=0,34)$, numa escala de 1 a 4 (Quadro 2).

A comparação emparelhada dos valores médios das respostas de pais e filhos sobre a percepção física, permite verificar que as crianças, independentemente do grau de comprometimento motor que patentearam (i.e., grupo I, II ou III), apresentaram valores médios relativos à competência física percebida sempre superiores (entre $2,79, \mathrm{dp}=0,38$ e 3,52 , $\mathrm{dp}=0,34$ ) aos apontados pelos seus pais (entre 1,68, $\mathrm{dp}=0,33$ até $2,53, \mathrm{dp}=0,52$ ). Em ambos os casos, observou-se que os valores médios de percepção de competência física encontrados, diminuíram significativamente à medida que o comprometimento 
Quadro 2. Média (desvio padrão] da percepção de competência motora pelas crianças e seus pais [escala EPPCASCPPp] por grupos relativos à avaliação da função motora global (TMFM-66).

\begin{tabular}{|c|c|c|c|}
\hline & Percepção de competência física das Crianças & Percepção de competência física pelos pais & \\
\hline & Média $[d p]$ & Média $[d p]$ & $p^{*}$ \\
\hline Grupo I & $3,52(0,34)$ & $2,53(0,52)$ & $<0,001$ \\
\hline Grupo II & $3,34(0,42)$ & $2,10(0,46)$ & $<0,001$ \\
\hline Grupo III & $2,79(0,38)$ & $1,68(0,33)$ & $<0,001$ \\
\hline$p^{* *}$ & $<0,001$ & $<0,001$ & \\
\hline
\end{tabular}

Quadro 3. Média (desvio padrão) da percepção de competência física pelas crianças (escala EPPCASCPCp) por grupos relativos à função motora (TMFM-66) e de acordo com características das crianças.

\begin{tabular}{|c|c|c|c|c|c|}
\hline & & Grupo I & Grupo II & Grupo III & \\
\hline & & Média (dp) & Média (dp) & Média (dp) & $p$ \\
\hline \multicolumn{6}{|l|}{ Sexo } \\
\hline Feminino & & $3,45(0,43)$ & $3,20(0,32)$ & $2,86(0,44)$ & 0,018 \\
\hline \multirow[t]{2}{*}{ Masculino } & & $3,55(0,30)$ & $3,48(0,47)$ & $2,70(0,27)$ & $<0,001$ \\
\hline & $p$ & 0,514 & 0,128 & 0,422 & \\
\hline \multicolumn{6}{|l|}{ Idade } \\
\hline$<$ Panos & & $3,50(0,42)$ & $3,49(0,38)$ & $2,94(0,48)$ & 0,041 \\
\hline \multirow[t]{2}{*}{$\geq 7$ anos } & & $3,53(0,30)$ & $2,96(0,21)$ & $2,66(0,28)$ & $<0,001$ \\
\hline & $p$ & 0,815 & 0,055 & 0,181 & \\
\hline \multicolumn{6}{|l|}{ Área de residência } \\
\hline Rural & & $3,53(0,35)$ & $3,45(0,43)$ & $2,71(0,27)$ & $<0,001$ \\
\hline \multirow[t]{2}{*}{ Urbana } & & $3,53(0,35)$ & $3,23(0,38)$ & $3,05(0,57)$ & 0,049 \\
\hline & $p$ & 0,846 & 0,174 & 0,177 & \\
\hline \multicolumn{6}{|l|}{ Escolaridade dos Pais } \\
\hline Até $9^{\circ}$ ano & & $3,50(0,35)$ & $3,31(0,40)$ & $2,81(0,46)$ & 0,001 \\
\hline \multirow[t]{2}{*}{$>9^{\circ}$ ano } & & $3,60(0,33)$ & $3,39(0,44)$ & $2,77(0,21)$ & 0,002 \\
\hline & $p$ & 0,399 & 0,621 & 0,869 & \\
\hline \multicolumn{6}{|l|}{ Capacidade de locomoção } \\
\hline Com marcha independente & & $3,52(0,34)$ & $3,04(0,34)$ & - & 0,004 \\
\hline \multirow[t]{2}{*}{ Sem marcha independente } & & - & $3,53(0,35)$ & $2,79(0,38)$ & 0,001 \\
\hline & $p$ & - & 0,022 & - & \\
\hline \multicolumn{6}{|l|}{ Classificação topográfica } \\
\hline Tetraplegia & & $3,52(0,46)$ & $3,22(0,43)$ & $2,72(0,49)$ & 0,019 \\
\hline Diplegia & & $3,37(0,30)$ & $3,48(0,39)$ & $2,86(0,22)$ & 0,002 \\
\hline \multirow[t]{2}{*}{ Hemiplegia } & & $3,60(0,30)$ & $2,90(0,00)$ & - & 0,026 \\
\hline & $p$ & 0,136 & 0,142 & 0,164 & \\
\hline \multicolumn{6}{|l|}{ Actividade desportiva } \\
\hline Com prática & & $3,55(0,37)$ & $3,41(0,47)$ & $2,76(0,29)$ & 0,001 \\
\hline \multirow[t]{2}{*}{ Sem prática } & & $3,44(0,26)$ & $3,29(0,39)$ & $2,82(0,46)$ & 0,014 \\
\hline & $p$ & 0,145 & 0,434 & 0,873 & \\
\hline
\end{tabular}


Quadro 4. Média (desvio padrão) da percepção de competência física das crianças pelos pais (escala EPPCASCPCp) por grupos relativos à função motora (TMFM-66) e de acordo com características das crianças.

\begin{tabular}{|c|c|c|c|c|c|}
\hline & & Grupo I & Grupo II & Grupo III & \\
\hline & & Média (dp) & Média (dp] & Média (dp) & $p$ \\
\hline \multicolumn{6}{|l|}{ Sexo } \\
\hline Feminino & & $2,68(0,49)$ & $1,90(0,42)$ & $1,73(0,40)$ & $<0,001$ \\
\hline \multirow[t]{2}{*}{ Masculino } & & $2,47(0,52)$ & $2,28(0,43)$ & $1,61(0,24)$ & 0,001 \\
\hline & $\mathrm{p}$ & 0,253 & 0,049 & 0,524 & \\
\hline \multicolumn{6}{|l|}{ Idade } \\
\hline$<$ Panos & & $2,50(0,45)$ & $2,12(0,50)$ & $1,54(0,35)$ & 0,002 \\
\hline \multirow[t]{2}{*}{$\geq 7$ anos } & & $2,55(0,55)$ & $2,03[0,41]$ & $1,79(0,29)$ & 0,001 \\
\hline & $\mathrm{p}$ & 0,749 & 0,581 & 0,184 & \\
\hline \multicolumn{6}{|l|}{ Área de residência } \\
\hline Rural & & $2,61(0,45)$ & $2,23(0,36)$ & $1,66(0,29)$ & $<0,001$ \\
\hline \multirow[t]{2}{*}{ Urbana } & & $2,40(0,62)$ & $1,95(0,53)$ & $1,75(0,48)$ & 0,105 \\
\hline & $\mathrm{p}$ & 0,201 & 0,163 & 0,761 & \\
\hline \multicolumn{6}{|l|}{ Escolaridade dos Pais } \\
\hline Até $9^{\circ}$ ano & & $2,52(0,55)$ & $1,93(0,42)$ & $1,68(0,37)$ & $<0,001$ \\
\hline \multirow[t]{2}{*}{$>9^{\circ}$ ano } & & $2,56(0,44)$ & $2,29(0,44)$ & $1,68(0,29)$ & 0,005 \\
\hline & $\mathrm{p}$ & 0,880 & 0,049 & 0,870 & \\
\hline \multicolumn{6}{|l|}{ Capacidade de locomoção } \\
\hline Com marcha independente & & $2,55(0,51)$ & $2,11(0,39)$ & - & 0,034 \\
\hline \multirow[t]{2}{*}{ Sem marcha independente } & & $1,80(-)$ & $2,09(0,52)$ & $1,68(0,33)$ & 0,090 \\
\hline & $\mathrm{p}$ & 0,132 & 0,901 & - & \\
\hline \multicolumn{6}{|l|}{ Classificação topográfica } \\
\hline Tetraplegia & & $2,65(0,53)$ & $2,16(0,39)$ & $1,46(0,28)$ & 0,001 \\
\hline Diplegia & & $2,24(0,48)$ & $2,09(0,47)$ & $1,90(0,22]$ & 0,231 \\
\hline \multirow[t]{2}{*}{ Hemiplegia } & & $2,63(0,51)$ & $2,00(0,85)$ & - & 0,267 \\
\hline & $\mathrm{p}$ & 0,081 & 0,988 & 0,005 & \\
\hline \multicolumn{6}{|l|}{ Actividade desportiva } \\
\hline Com prática & & $2,64(0,50)$ & $2,30(0,48)$ & $1,80(0,23)$ & $<0,001$ \\
\hline \multirow[t]{2}{*}{ Sem prática } & & $2,28(0,53)$ & $1,92(0,37)$ & $1,56(0,39)$ & 0,009 \\
\hline & $\mathrm{p}$ & 0,054 & 0,037 & 0,170 & \\
\hline
\end{tabular}

Valor de $p$ recorrendo aos testes de Mann-Whitney e de Kruskal-Wallis

Quadro 5. Coeficientes de correlação de Spearman entre a função motora e a percepção de competência física avaliada pelas crianças e pelos pais.

\begin{tabular}{|c|c|c|c|}
\hline & Função motora & $\begin{array}{l}\text { *Percepção Competência } \\
\text { Física das Crianças** }\end{array}$ & $\begin{array}{l}\text { Percepção Competência Física } \\
\text { das Crianças avaliada pelos pais** }\end{array}$ \\
\hline Função motora & - & $0,503(* * *)$ & $0,621\left(^{* * *}\right]$ \\
\hline Percepção Competência Física das Crianças & & - & $0,393[* * *]$ \\
\hline
\end{tabular}

*avaliada através da TMFM-66; **avaliada através da EPPCASCPCp; ${ }^{* * *} p<0,001$. 
motor evidenciado pelas crianças aumentava. Os valores médios de percepção de competência física avaliados pelas crianças e pelos pais foram também comparados atendendo não só aos diferentes grupos de função motora considerados (i.e., Grupos I, II e III) como também às diferentes características das crianças, anteriormente descritas (Quadros $3 \mathrm{e}$ 4). A diminuição dos valores médios de percepção física avaliada pelas crianças de acordo com a diminuição da função motora, já descrita, manteve-se independentemente do sexo, idade, local de residência, escolaridade dos seus pais, classificação topográfica, capacidade de locomoção, e ainda da prática desportiva. Dentro de cada um dos grupos de função motora considerados, as médias de competência física percebida não variaram significativamente de acordo com as características estudadas, com a excepção da variável capacidade de locomoção no grupo II da função motora (Quadro 3).

Verificou-se a mesma tendência decrescente nos valores médios de percepção que os pais tem da competência física das crianças, de acordo com a capacidade física avaliada pela função motora (TMFM-66), para todas as características avaliadas, excepto nas crianças residentes em áreas rurais e nas crianças diplégicas e hemiplégicas onde as diferenças não atingiram significado estatístico (Quadro 4). Considerando a comparação dentro de cada grupo de função motora, apenas se verificaram diferenças significativas nas médias de percepção de competência física pelos pais de acordo com a classificação topográfica, nas crianças do grupo III. A média de percepção de competência física pelos pais foi significativamente superior nas crianças com diplegia quando comparadas com as tetraplégicas $(1,90, \mathrm{dp}=0,22 \mathrm{vs}$. $1,46, d p=0,28 ; p=0,005)$.

Também nas crianças do grupo II a média de percepção de competência física pelos pais foi significativamente superior nas crianças praticantes de actividade física quando comparadas com as que não praticavam $(2,30, \mathrm{dp}=0,48$ vs. $1,92, \mathrm{dp}=0,37 ; \mathrm{p}=0,037)$. No quadro 5 encontram-se descritos os coeficientes de correlação de Spearman entre a função motora e a percepção de competência física avaliada pelas crianças e pelos pais. As correlações encontradas entre os diferentes instrumentos foram moderadas. A correlação mais elevada foi encontrada entre a função motora e a percepção de competência física avaliada pelos pais $(r=0,621)$ e a mais baixa correlação foi encontrada entre a avaliação da competência percebida pelas crianças e pelos pais $(r=0,393)$.

Dada a diferença do âmbito de variação dos scores finais dos instrumentos utilizados (TMFM-66 e PSPCSACCPp), optou-se por distribuir os participantes de acordo com a distribuição por tercis avaliando a concordância de classificação de acordo com subdivisão em tercis, correspondendo o $1^{\circ}$ tercil a um grau superior de comprometimento motor. A percentagem de concordância observada entre a classificação de acordo com a função motora e as percepções de competência física avaliadas pelas crianças e pelos pais foram de 49,4 e 50,6, respectivamente. Os correspondentes valores de coeficiente Kappa foram de 0,241 e 0,259. A percentagem de concordância mais elevada foi observada nos grupos pertencentes ao terceiro tercil ou seja nas crianças com maior capacidade motora (Quadro 6). A discordância de classificação extrema, ou seja em tercis opostos, foi de $12,3 \%$ e $7,4 \%$, respectivamente. Foi ainda avaliada a concordância entre a classificação por tercis da competência física percebida das crianças com a avaliada pelos seus pais. A concordância observada foi de $48,1 \%$ e o coeficiente Kappa de 0,219 (Quadro 7). A discordância de classificação extrema foi de $13,6 \%$.

\section{DISCUSSÃO}

A análise da distribuição percentual da amostra pelos grupos de função motora, definidos a partir da avaliação aferida com base na TMFM-66. (i.e., grupos I, II e III), e de acordo com um conjunto de características demográficas, sociais, clínicas e comportamentais das crianças avaliadas, permitiu constatar que a mesma distribuição apenas variou significativamente de acordo com a capacidade de locomoção das mesmas. Estes resultados estão de acordo com o esperado, i.é., uma percentagem de crianças não ambulatórias (i.e., crianças sem marcha independente) superior nos grupos II e III, onde efectivamente o grau de comprometimento era mais acentuado e nenhuma criança ambulatória no grupo III. Em concordância com os resultados de um estudo de Russell e colaboradores ${ }^{(46)}$, nas crianças com uma função motora global superior a $89 \%$, ou seja, as do 
Quadro 6. Concordância de classificação por tercis da função motora e da competência física percebida das crianças e dos pais.

\begin{tabular}{|c|c|c|c|c|c|c|c|}
\hline & & \multicolumn{3}{|c|}{$\begin{array}{l}\text { Percepção Competência } \\
\text { Física das Crianças** }\end{array}$} & \multicolumn{3}{|c|}{$\begin{array}{l}\text { Percepção Competência Física } \\
\text { das Crianças avaliada pelos pais** }\end{array}$} \\
\hline & & $1^{\circ}$ tercil & $2^{\circ}$ tercil & $3^{\circ}$ tercil & $1^{\circ}$ tercil & $2^{\circ}$ tercil & $3^{\circ}$ tercil \\
\hline \multirow[t]{4}{*}{ Função motora* } & $1^{0}$ tercil & $12(44,4 \%)$ & $9(33,3 \%)$ & $6(22,2 \%)$ & $15(55,6 \%)$ & $8(29,6 \%)$ & $4(14,8 \%)$ \\
\hline & $2^{\circ}$ tercil & $7(25,9 \%)$ & $12(44,4 \%)$ & $8(29,6 \%)$ & $9(33,3 \%)$ & $>(25,9 \%)$ & $11(40,7 \%)$ \\
\hline & $3^{\circ}$ tercil & $4(14,8 \%)$ & $>(25,9 \%)$ & $16(59,3 \%)$ & $2(7,4 \%)$ & $6(22,2 \%)$ & $19(70,4 \%)$ \\
\hline & & \multicolumn{3}{|c|}{$\begin{array}{c}\text { Concordância observada }=49,4 \% \\
\text { Kappa }=0,241\end{array}$} & \multicolumn{3}{|c|}{$\begin{array}{c}\text { Concordância observada=50,6\% } \\
\qquad \text { Kappa }=0,259\end{array}$} \\
\hline
\end{tabular}

*avaliada através da TMFM-66; **avaliada através da EPPCASCPCp

Quadro 7. Concordância de classificação por tercis da competência física percebida das crianças e dos pais.

\begin{tabular}{|c|c|c|c|c|}
\hline & & \multicolumn{3}{|c|}{$\begin{array}{l}\text { Percepção Competência Física } \\
\text { das Crianças avaliada pelos pais* }\end{array}$} \\
\hline & & $1^{0}$ tercil & $2^{0}$ tercil & $3^{\circ}$ tercil \\
\hline \multirow[t]{4}{*}{ Percepção Competência Física das Crianças* } & $1^{\circ}$ tercil & $14(60,9 \%)$ & $5(21,7 \%)$ & $4(17,4 \%)$ \\
\hline & $2^{0}$ tercil & $5(17,9 \%)$ & $9(32,1 \%)$ & $14(50,0 \%)$ \\
\hline & $3^{\circ}$ tercil & $7(23,3 \%)$ & $>(23,3 \%)$ & $16(53,3 \%)$ \\
\hline & \multicolumn{4}{|c|}{$\begin{array}{l}\text { Concordância observada }=48,1 \% \\
\qquad K a p p a=0,219\end{array}$} \\
\hline
\end{tabular}

*avaliada através da EPPCASCPCP.

grupo I, esperar-se-ia um desempenho motor sem grandes dificuldades e, como tal, uma natural capacidade de marcha independente. No extremo oposto, nas crianças do grupo III, isto é, com uma função motora global inferior a $61 \%$, prever-se-ia uma maior dificuldade na realização de várias situações, nomeadamente a sua capacidade de locomoção. No presente estudo as crianças, independentemente do seu comprometimento motor, apresentaram valores de percepção de competência acima da média, ou seja, valores médios de $3,34(\mathrm{dp}=0,46)$, numa escala de 1 a 4 . Estudos realizados neste âmbito, com outras populações com necessidades especiais, nomeadamente de Dunn e Watkinsson(16), em crianças com problemas de coordenação, Glenn \& Cunningham(21) em crianças com Síndrome de Down, e Vaughn e colaboradores(58) em crianças com dificuldades de aprendizagem, apoiam os resultados deste estudo, confirmando a tendência das crianças se sentirem competentes fisicamente. Os valores encontrados por Julie Čurdová e colaboradores(15) e Vanessa Scholtes e colaboradores ${ }^{(48)}$, em pesquisas realizadas em crianças com $\mathrm{PC}$, pertencentes ao mesmo escalão etário por nós considerado e usando a mesma escala, confirmaram o facto destas se sentirem competentes em termos motores, encontrando valores médios de 3,42 (dp=0,87) e 3,45 $(d p=0,45)$, respectivamente.

Verificamos que a percepção de competência física das crianças é superior à percepção dos seus pais. Tal como postulado por alguns autores $(15,43,59)$, parece pois, existir um padrão de resposta nas crianças, neste caso com PC, ao referirem que devemos ter em conta que nestas idades, na tentativa de minimizarem eventuais rejeições, principalmente da parte das pessoas que lhes são mais significativas, possam sobrestimar as suas competências, evidenciando mesmo uma tendência para confundirem a realidade com o seu desejo de serem competentes $(24,55)$. Esta situação, segundo Harter(27), induz as crianças a responderem de acordo com aquilo que entendem ser socialmente mais aceitável e, como tal, a construírem julgamentos positivos e a sobrevalorizarem as suas competências. Cole e colaboradores(11) alertam 
ainda para a possibilidade de, muitas vezes, esta situação poder ser uma consequência da interiorização de padrões dos agentes socializadores, nomeadamente pais e professores.

Um estudo de Hoza e colaboradores(29), envolvendo a mesma metodologia, mas em crianças com défice de atenção e síndrome de hiperactividade, revelou a mesma tendência de resposta. Segundo aqueles autores, estas diferenças aceitam-se se considerarmos que a maturidade, vivência e identificação com a realidade por parte dos adultos, se traduzem numa maior capacidade em percepcionarem a real competência das crianças.

Nesta situação, os pais parecem funcionar como um bom indicador externo das capacidades dos seus filhos, tal como referem Morris e colaboradores(36) num estudo em que utilizando o Gross Motor Function Classification System de ${ }^{(39)}$ onde foram avaliadas conjuntamente a capacidade de avaliação da função motora geral das crianças pelos pais e outros profissionais de saúde. Segundo este autor, os pais terão uma boa capacidade de avaliação dos seus filhos uma vez que, para além da situação específica avaliada, têm um conhecimento mais alargado das suas possibilidades resultante de um contacto mais frequente e num espectro alargado de situações, que lhe permitem uma avaliação mais consistente, contrariamente a outros profissionais que estão privados da observação de determinadas realizações das crianças em termos funcionais, nomeadamente em contexto familiar(39). Segundo Miller(35), a maioria dos pais ao tomar consciência da patologia do seu filho e, como tal, das suas dificuldades, sente necessidade de desenvolver um aprofundado conhecimento acerca da mesma, tentando deste modo uma mais efectiva avaliação da situação que permita uma melhor capacidade para interagir com a criança que lhe confira mais segurança e aptidão para a ajudar. Sollerhed e colaboradores ${ }^{(52)}$, por seu lado, sublinham o facto desta tendência de sobrevalorização diminuir à medida que avançamos no escalão etário, tal como observado neste estudo, onde se verificaram, valores médios mais elevados de competência física percebida no grupo das crianças mais novas comparativamente às mais velhas, embora sem atingir significado estatístico. Susan Harter(26), Horn \& Hasbrook (28) e Rudisill e colaboradores(43), haviam já alertado para o facto da capacidade de autopercepção das crianças se diferenciar com a idade, podendo as idades mais baixas, de acordo com Jacobs e colaboradores(30) funcionar como predictoras de valores de percepção mais elevados. Esta diferenciação, tal com defendem outros autores $(37,41,48)$, parece sugerir uma evolução na qualidade da avaliação das crianças, à medida que a idade delas aumenta, tornando-se cada vez mais próxima do real.

Considerando ainda a amostra total, verificou-se que o valor médio de percepção de competência física foi mais elevado nos rapazes $(3,42$ vs. 3,$21 ; \mathrm{p}=0,048)$. Como referem Anderssen \& Wold(1) e Crocker e colaboradores $^{(14)}$, os rapazes recebem socialmente mais estímulos para serem fisicamente activos, valorizando mais a sua participação em actividades físicas. Por outro lado, essa maior solicitação também os confronta com uma maior probabilidade de insucesso, o que de certa forma permite explicar estes resultados, pois os feedbacks daquelas participações nem sempre serão positivos.

Apesar de ser necessária alguma prudência na interpretação da avaliação pelas crianças, não apenas pela tendência já referida anteriormente de sobreavaliação, mas também, e na linha do que defende Harter(27), pelo facto dessa sobrestimação poder ser reflexo de desejo de competência e não de competência efectiva, essa estimativa é proporcional ao grau de comprometimento motor o que apoia o interesse da sua utilização. De facto, os valores médios de percepção de competência física das crianças e dos seus pais, observados neste estudo, revelam que houve um decréscimo à medida que aumentava o grau de comprometimento motor, avaliado pela escala de função motora.

Tendo em atenção que a PC também varia na extensão das suas consequências $(4,6)$ parece-nos consensual aceitar que as crianças com um nível de comprometimento maior se sintam menos competentes em termos motores, reflectindo-se isso, em diferenças na sua capacidade e qualidade de percepção de competência física.

A inexistência de estudos, à luz do nosso conhecimento, que tenham utilizado especificamente estes dois instrumentos (EPPCASCPCp e TMFM-66) como forma de comparar medidas de percepção de competência física com medidas mais objectivas que 
quantificam o grau de função motora em crianças com PC, dificulta a discussão directa dos nossos resultados. Contudo, alguns autores utilizaram comparações semelhantes pelo recurso a outros instrumentos de avaliação, tanto em crianças com deficiência como em crianças sem qualquer patologia. Welk e colaboradores(61), utilizando o Physical SelfPerception Profile e o Fitnessgram Fitness Profile, em crianças ligeiramente mais velhas que as do nosso estudo (i.e., entre os 9 e 10 anos), avaliaram a relação entre a sua competência física percebida e a função motora real, tendo confirmado a existência de relação entre a auto-percepção física e a função motora real das mesmas. Num outro estudo, envolvendo crianças entre os 9 e 11 anos, Rudisill e colaboradores(43), utilizando a Motor Perceived Competence Scale ${ }^{(43)}$, analisaram a relação entre a competência motora real e percebida das crianças e concluíram que, neste escalão etário, elas manifestavam uma capacidade relativamente satisfatória na avaliação que faziam da sua competência motora $(r=0,33$ quando considerado o trem inferior e $r=0,35$ para o trem superior).

Pelo contrário, os resultados de um estudo com crianças, realizado na Estónia por Raudsepp \& Liblik(42), também avaliando a relação entre competência motora real e percebida, mostraram que as crianças manifestavam alguma dificuldade em perceber a sua competência, alertando no entanto, para o facto de poucos estudos terem sido efectuados em países de língua não inglesa. Estes autores, corroborando da opinião de Hager e colaboradores(23), sublinham a importância de um certo efeito cultural sobre os constructos das autopercepções físicas, resultando não apenas em diferentes estruturas factoriais como ainda em diferentes associações com o desempenho.

Não obstante termos verificado correlações satisfatórias entre a avaliação da percepção de competência física e da função motora real, a concordância de classificação por tercis foi genericamente baixa, sendo superior nas crianças com grau de comprometimento motor menor. Isto sugere que a sobrevalorização da competência seja proporcionalmente superior nos indivíduos com grau de função motora mais agravado. Curdová e colaboradores(15), sublinham o facto das crianças sem marcha independente, tenderem a comparar-se com os seus pares sem deficiência ou com deficiência mas independentes na marcha, levando a percepções de competência mais baixas. Apesar da influência de factores como o sexo e a idade na avaliação de percepção de competência físi$\mathrm{ca}$, descrita por diversos autores anteriormente referidos $(5,7,18,28,37,41,48)$, na presente análise, a tendência do decréscimo dos valores relativos à percepção de competência física, à medida que diminui a função motora, foi independente de características como o sexo, a idade, a área de residência, a escolaridade dos pais ou a prática de actividade desportiva. Podemos admitir que o reduzido tamanho amostral resultante da divisão por grupos, possa influenciar a possibilidade de encontrar diferenças significativas. A realização de estudos porventura envolvendo maior tamanho amostral poderia contribuir para o melhor esclarecimento destes aspectos. Como refere Fox ${ }^{(20)}$, é necessário garantir mais investigação nestes domínios em grupos específicos e de diferentes culturas.

Em conclusão, os valores médios das respostas relativos à EPPCASCPCp (subescala de percepção de competência física), atribuídos pelas crianças e pelos seus pais revelaram três aspectos fundamentais a considerar: i) de uma forma geral, numa escala de 1 a 4 as crianças apresentaram valores de percepção de competência acima da média, independentemente do seu comprometimento motor; ii) a avaliação que as crianças fazem da sua competência física é superior à que lhes foi atribuída pelos seus pais; iii) os valores médios de competência física percepcionados pelas crianças e pelos pais, decrescem à medida que aumenta o grau de comprometimento motor avaliado pela escala de função motora, o que apoia a utilização da da EPPCASCPCp para avaliação de um indicador indirecto de função motora em crianças com PC.

\section{CORRESPONDÊNCIA}

\section{Rui Corredeira}

Gabinete de Actividade Física Adaptada

Faculdade de Desporto, Universidade do Porto

Rua Dr. Plácido Costa, 91

4200-450 Porto

E-mail: rcorredeira@fade.up.pt 


\section{REFERÊNCIAS}

1. Anderssen N, Wold B (1992). Parental and peer influences on leisure-time physical activity in young adolescents. Research Quarterly for Exercise and Sport 63: 341-348.

2. Andrada MG, Gimenez JP (1991). Teste de Medida das Funções Motoras (TMFM) - Guia do Utilizador. Adaptação do Gross Motor Function Measure de Russell e colaboradores (1989). Centro de Paralisia Cerebral Calouste Gulbenkian. Lisboa.

3. Bandura A (1982). Self-efficacy mechanism in human agency. American Psychologist 37: 122-147.

4. Bax M, Goldstein M, Rosenbaum P, Levinton A, Paneth N (2005). Proposed definition and classification of Cerebral Palsy: Introduction. Developmental Medicine \& Child Neurology 47: 571- 576.

5. Biddle S, Armstrong N (1992). Children's physical activity: An exploratory study of psychological correlates. Social Science and Medicine 34: 325-331.

6. Bobath K (1984). Uma base neurofisiológica para o tratamento da Paralisia Cerebral. S.Paulo: Manole.

7. Bortoli 1, Robazza C (1997). Italian Version of the Perceived Physical Ability Scale. Perceptual and Motor Skills 85: $187-192$.

8. Burns RB (1979). The self-concept: Theory, measurement, development and behaviour. London: Longman.

9. Calsyn R, Kenny D (1977). Self-concept of ability and perceived evaluation of others: Cause or effect of academic achievement? Journal of Educational Psychology 69: 136-145.

10. Chapman J (1988). Learning disabled children's self-concepts. Review of Educational Research 58: 347-371.

11. Cole D, Maxwell S, Martin J (1997). Reflected self-appraisals, strength and structure of the relation of teacher, peer and parent ratings to children's self-perceived competencies. Journal of Educational Psychology, 89 (1), 55-70.

12. Cooley EJ, Ayres R (1988). Self-concept and success-failure attributions of non-handicapped students with Learning Disabilities. Journal of Learning Disabilities 21: 174-178.

13. Corredeira R, Côrte-Real NJ, Dias C, Silva MA, Fonseca, AM (2007). Como avaliar a Percepção de Competência e Aceitação Social de Crianças com Paralisia Cerebral? Estudo inicial para a determinação das propriedades psicométricas da versão portuguesa da Dutch Pictorial Scale of Perceived Competence and Social Acceptance in Children with Cerebral Palsy. Revista Brasilera de Educação Especial (In press).

14. Crocker PR, Eklund RC, Kowalski KC (2000) Children's physical activity and physical self-perceptions. Journal of Sports Science 18: 383-94.

15. Curdová J, Vermeer A, Válková H (2001). Measuring perceived and social acceptance in young children with cerebral palsy: the construction of Czech Pictorial Scale. Gymnica 1(1): 27-36.

16. Dunn J, Watkinson E (1994). A study of the relationship between physical awkwardness and children's perceptions of physical competence. Adapted Physical Activity Quarterly 11: 275-283.

17. Ebbeck V, Weiss MR (1998). Determinants of children's self-esteem: Influence of perceived competence and affect. Pediatric Exercise Science 10: 285-298.

18. Eccles J, Wigfield A, Harold R (1993). Age and gender differences in children's self - and task perceptions during elementary school. Child Development 64: 830-47.
19. Faria L (2005). Desenvolvimento do auto-conceito físico nas crianças e nos adolescentes. Análise Psicológica 4: 361371.

20. Fox KR (2000). The effects of exercise on self-perceptions and self-esteem. In: SJH Biddle, KR Fox, SH Boutcher (eds). Physical activity and psychological well-being (pp. 88117). London: Routledge.

21. Glenn S, Cunningham C (2001). Evaluation of self by young people with Down Syndrome. International Journal of Disability, Development and Education 48: 163-177.

22. Goodway J, Rudisill M (1996). Influence of a motor skill intervention program on perceived competence of at-risk african-american pre-schoolers. Adapted Physical Activity Quarterly 13: 288-301.

23. Hagger M, Biddle SJ, Wang CK (2005). Physical SelfConcept in Adolescence: Generalizability of a Multidimensional, Hierarchical Model Across Gender and Grade. Educational and Psychology Measurement 65: 297-322.

24. Harter S (1982). The perceived competence scale for children. Child Development 53: 87-97.

25. Harter S, Pike R (1984). The Pictorial Scale of Perceived Competence and Social Acceptance for Young Children. Child Development 55: 1968-1982.

26. Harter S (1985). Competence as a dimension of self-evaluation: toward a comprehensive model of self-worth. In: Leahy R (ed.). The Development of the Self. (pp. 55-121). New York: Academic Press.

27. Harter S (1999). The Construction of the Self - A developmental Perspective. New York: The Guilford Press.

28. Horn T, Hasbrook C (1987). Psychological characteristics and the criteria children use for evaluation. Journal of Sport Psychology 9: 208-221.

29. Hoza B, Gerdes A, Hinshaw S, Arnold L (2004). Self-perceptions of competence in children with ADHD and comparison children. Journal of Consulting and Clinical Psychology 72: 382-391.

30. Jacobs JE, Lanza S, Osgood DW (2002). Changes in children's self-competence and values: gender and domain differences across grades one through twelve. Child Development 73: 509-27.

31. King G, Spetch J, Schultz I, Warr-Leeper G, Redekop W, Risebrough N (1997). Social skills training for withdraw unpopular children with physical disabilities - A preliminary evaluation. Rehabilitation Psychology 42: 47-60.

32. Klein S, Evans J (1998). Perceptions of competence and peer acceptance in young children with motor and learning difficulties. Physical and Occupational Therapy in Paediatrics: A Quarterly Journal of Development 18: 39-52.

33. Kunnen E (1992). Mastering (with) a handicap Development of task-attitudes in physically handicapped children. Groningen: Stitching Kinder studies.

34. Lerner RM (1991). Changing organism-context relations as a basic process of development: A developmental contextual perspective. Developmental Psychology 27: 27-32.

35. Miller G (2002). Paralisias Cerebrais: Uma visão geral. In: G Miller, GD Clark (eds). Paralisias Cerebrais - Causas, consequências e conduta (pp. 1-40). São Paulo: Manole.

36. Morris C, Kurinczuk JJ, Fitzpatrick R, Rosenbaum PL (2006). Who best to make the assessment? Professionals' and families' classifications of gross motor function in cerebral palsy are highly consistent. Archives of Disease in Childhood 91: 675-679. 
37. Nicholls J (1978). The development of the concepts of effort and ability, perception of academic attainment, and the understanding that difficult tasks require more ability. Child Development 49: 800-814.

38. Nicholls DS, Case-Smith J (1996). Reliability and validity of the Pediatric Evaluation of Disability Inventory. Pediatric Physical Therapy 8:15-24.

39. Palisano RJ, Rosenbaum PL, Walter SD, Russell DJ, Wood EP, Galuppi BE (1997). Development and reliability of a system to classify gross motor function in children with cerebral palsy. Development Medicine Child Neurology 39: 214-223.

40. Poole JR, Mathias K, Stratton, Richard K (1996). HigherSkilled and Lower-Skilled Children's Perceived Ability and Actual Performance with Kicking and Striking Tasks. The Physical Educator 53: 214-221.

41. Raudsepp L, Liblik R (2002). Relationship of perceived and actual motor competence in children. Perceptual and Motor Skills 94: 1059-1070.

42. Rodger S, Ziviani J, Watter P, Ozanne A, Woodyatt G, Springfield E (2003). Motor and functional skills of children with developmental coordination disorder: a pilot investigation of measurement issues. Human Movement Science 22: 461-478.

43. Rudisill ME, Mahar MT, Meaney KS (1993). The Relationship between Children's Perceived and Actual Motor Competence. Perceptual And Motor Skills 76: 895906.

44. Russell DJ, Rosembaum PL, Cadman DT, Gowland C, Hardy S, Jarvis S (1989). The Gross Motor Function Measure: a mean to evaluate the effects of physical therapy. Development Medicine Child Neurology 31: 341-52.

45. Russell DJ, Rosembaum PL, Avery LM, Lane M (2002). Gross Motor Function Measure (GMFM-66 \& GMFM-88) User's Manual. London: Mac Keith Press.

46. Russell DJ, Rosembaum PL, Raina PS, Walter SD, Palisano RJ (2000). Improved Scaling of the Gross Motor Function Measure for Children With Cerebral Palsy: Evidence of Reliability and Validity. Physical Therapy 80: 873-885.

47. Scholtes V, Vermeer A, Meek G (2002). Measuring perceived competence and social acceptance in children with cerebral palsy. European Journal of Special Needs Education 17: 77-87.

48. Schwarz D (1998). The Pictorial Scale of Perceived Competence and Social Acceptance for Children with Cerebral Palsy: a reliability and validation study of a German version. Dissertação de Mestrado. Freie Universitat Berlin: Berlin.

49. Shavelson RJ, Bolus R (1982). Self-concept: the interplay of theory and methods. Journal of Educational Psychology 74: 3-17.

50. Silon E, Harter S (1985). Perceived competence, motivational orientation and anxiety in mainstreamed and selfcontained educable mentally retarded children. Journal of Educational Psychology 77: 217-230.

51. Sollerhed A-C, Apitzsch E, Råstam L, Ejlertsson G (2007). Factors associated with young children's self-perceived physical competence and self-reported physical activity. Health Education Research 12: 3-12.

52. Sternberg RJ (1993). The Princess grows up: A satiric fairy tale about intellectual development. In: RJ Sternberg, CA Berg (eds.). Intellectual development (pp. 381-394). Cambridge: Cambridge University Press.
53. Stipek DJ (1981). Children's perceptions of their own and their classmates' ability. Journal of Educational Psychology 73: 404-410.

54. Stipek DJ, Kowalski PS (1989). Learned helplessness in task-oriented versus performance-oriented testing conditions. Journal of Educational Psychology 81: 384-91.

55. Stone CA, May AJ (2002). The accuracy of academic selfperceptions in adolescents with learning disabilities. Journal of Learning Disabilities 35: 370-383.

56. Ulrich BD (1987). Perceptions of Physical Competente, Motor Competente, and Participation in Organized Sport: their interrelationship in young children. Research Quarterly for Exercise and Sport 58: 57-67.

57. Vaughn S, Hoga A, Kouzekanani K, Shapiro S (1990). Peer Acceptance, Self-Perceptions, and Social Skills of Learning Disabled Students Prior to Identification. Journal of Educational Psychology 82: 101-106.

58. Vermeer A, Lanen W, Hendriksen J, Speth L, Mulderij K (1994). Measuring perceived competence in children with cerebral palsy. In: JHA van Rossum, JI Laszlo (eds.). Motor Development: Aspects of normal and delayed development (pp. 133-144). Amsterdam: VU University Press.

59. Vermeer A, Veenhof C (1997). The Dutch Pictorial Scale of Perceived Competence and Social Acceptance for Children with Cerebral Palsy: a reliability and validation study. Dissertação de Mestrado. Vrije Universiteit Amsterdam: Amsterdam.

60. Welk GJ, Corbin CB, Dowell MN, Harris H (1996). The Relationship Between Physical Self-Perception and Physical Fitness in: Children Research Quarterly for Exercise and Sport 67: Supplement p. A-102.

61. Yun J, Ulrich D (1997). Perceived and actual physical competence in children with mild mental retardation. Adapted Physical Activity Quarterly 14: 285-297. 